\title{
Research on the senescence of human skin fibroblasts induced by ultraviolet b and its mechanism.
}

\author{
Xiaoqing Xiao, Minghuan Huang, Chunyan Fan, Fuguo Zuo* \\ Department of Dermatology, Shanghai East Hospital, Tongji University School of Medicine, PR China
}

\begin{abstract}
Objective: To discuss the effect of ultraviolet B (UVB) on inducing the senescence of human skin fibroblasts and investigate its mechanism.

Methods: The tissue explants method was used to isolate and culture the primary human skin fibroblasts, and after the observation of changes in cell morphology and $\beta$-galactosidase ( $\beta$-gal) cell senescence staining via irradiation with different doses of UVB, the single irradiation dose of UVB in the induction of senescence of skin fibroblasts in subsequent experiment was determined. The cells and supernatant were collected at different time points $(12,24,48$ and 72 h) after irradiation with established doses of UVB. Western blotting was performed to detect the expression of marker P16 protein in induced senescent cells, and the expression of type I collagen, type III collagen, matrix metalloproteinase (MMP)-1 and MMP-3 of senescent cells was detected via ELISA.

Results: According to Western blotting analysis, the expression of P16 protein in induced senescent skin fibroblasts was increased with the prolongation of irradiation time of UVB. ELISA showed that the expression of type I and III collagen was significantly reduced with the prolongation of irradiation time of UVB, while the expression of MMP1 and MMP3 was significantly increased.

Conclusion: UVB irradiation can induce the senescence of human skin fibroblasts, whose mechanism may be related to the decrease of collagen synthesis and the increase of collagen degradation.
\end{abstract}

Keywords: Human skin fibroblasts, Senescence, Collagen, Matrix metalloproteinase, Ultraviolet B.

Accepted on October 28, 2017

\section{Introduction}

At present, senescence is an irreversible biological phenomenon [1]. Skin senescence is part of the body senescence, which is manifested as skin relaxation, thinning, wrinkle formation and gradual increase and deepening in wrinkle [2], and it is the most intuitive external manifestation of the body senescence. Studies [3] have shown that the skin senescence is related to the decrease in skin fibroblast proliferation and dermal collagen content. At present, the specific role and mechanism of skin fibroblasts in the skin senescence process remain unclear [4]. In this study, the senescence of skin fibroblasts was induced via ultraviolet $\mathrm{B}$ (UVB), the expression of senescence-related protein P16 and changes in synthesis and secretion of type I and III collagen and matrix metalloproteinase (MMP) 1 and MMP3 were observed, and its possible mechanism was investigated.

\section{Materials and Methods}

\section{Main reagents and instruments}

High glucose DMED, fetal bovine serum and 0.25\% trypsin 0.02 EDTA (Gibco); $\beta$-galactosidase $(\beta$-gal) cell senescence staining kit, and $2 \times$ SDS protein lysis solution (Beyotime); type I and III collagen and MMP1 and MMP3 ELISA kit (BD); Scientz03-II UV cross-linker (Ningbo Xinzhi Biotechnology Co., Ltd.).

\section{Determination of the single irradiation dose of $U V B$ - induced cell senescence via preliminary experiment}

Separation and culture of human skin fibroblasts [5]: The excess foreskin tissues resected via urinary surgery (with informed consent of patients' guardians) were collected from the Third People's Hospital affiliated to School of Medicine, Shanghai Jiaotong University, and the subcutaneous fat and blood vessels were removed, and the foreskin fibroblasts were obtained using the tissue explants method. The tissues were cultured in the high-glucose DMED with $10 \%$ fetal bovine serum, and when the cells grew until $90 \%$, they were digested via $0.25 \%$ trypsin- $0.02 \%$ EDTA, followed by passage culture. The third-generation cells were taken for experiments.

Skin fibroblast senescence induced by UVB: The thirdgeneration human skin fibroblasts were irradiated with UVB (312 nm) using the UV cross-linker; the irradiation dose was 0 (blank control), 10, 20, 30, 40, 50, 60, 70 and $80 \mathrm{~mJ} / \mathrm{cm}^{2}$; after $48 \mathrm{~h}$, they were re-inoculated in the 6 -well plate $(700$ cells/ $\mathrm{cm}^{2}$ ); after $24 \mathrm{~h}$, the $\beta$-gal cell senescence staining kit was used 
to detect the cell senescence; according to the cell morphological changes and $\beta$-gal staining results, the single irradiation dose of UVB was determined.

\section{Detection of P16 protein expression via Western blotting}

\section{Cell grouping and induction of senescence:}

1) Irradiation group: The third generation of human skin fibroblasts were taken and inoculated into the culture dish with a $10 \mathrm{~cm}$ diameter $\left(1 \times 10^{6} / \mathrm{dish}\right)$; after the cell fusion of $80 \%$, they were irradiated with the single dose of UVB determined in the preliminary experiment. 2) Control group: It received no UVB irradiation, and other treatment was the same as that in irradiation group.
Western blotting: The cells were collected since the UVB irradiation (at $0 \mathrm{~h}$ ) and at 12, 24, 48 and $72 \mathrm{~h}$ after irradiation. The cells were split via $2 \times$ SDS protein lysate and the protein was collected. The cells were soaked in boiling water for 10 min, followed by $30 \%$ SDS PAGE (1:1); then the cells were sealed via 5\% skim milk for $2 \mathrm{~h}$, incubated using rabbit antihuman P16 (1:1000) at $4{ }^{\circ} \mathrm{C}$ overnight, washed for three times with TBS (10 min per time), and then the mouse anti-rabbit secondary antibody $(1: 2000)$ was added for incubation at room temperature for $2 \mathrm{~h}$, followed by color development via ECL kit, image scanning via image analyzer, and semi-quantitative analysis with GAPDH as the internal control.
$\mathbf{A}$

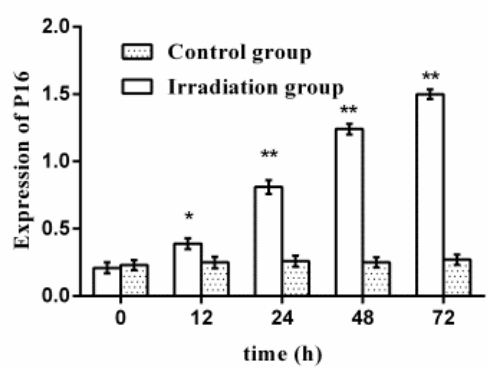

B

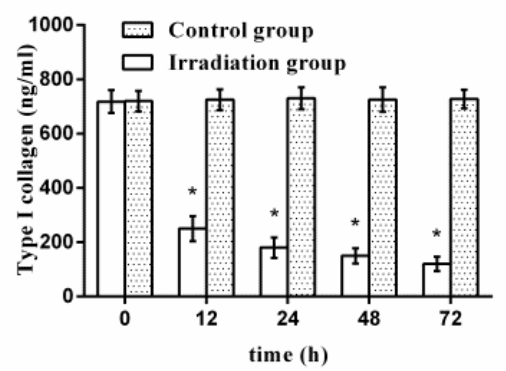

C

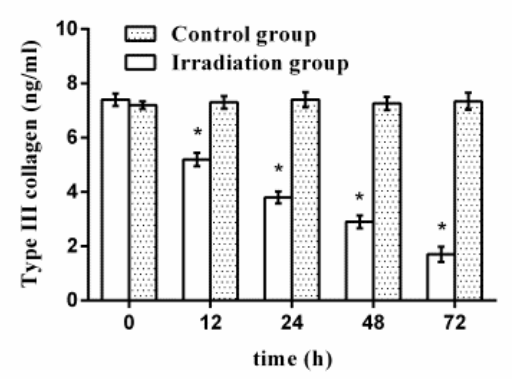

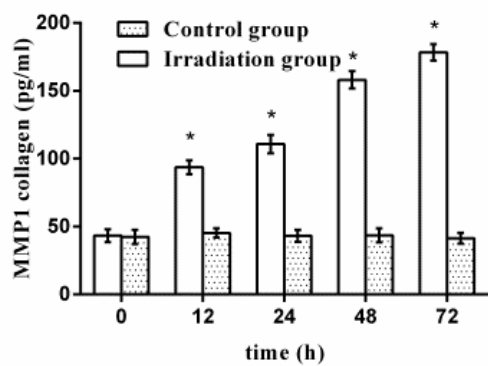

E

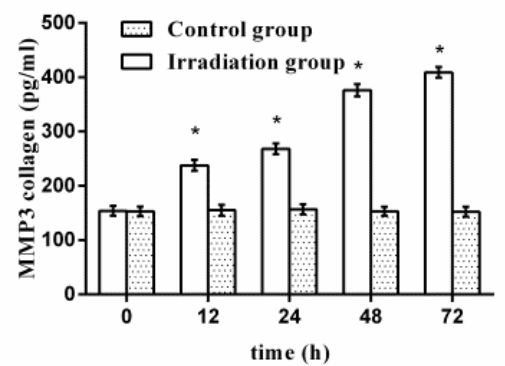

Figure 1. A) Expression of P16 protein in skin fibroblasts detected by Western blotting 1-5: at 0, 12, 24, 48 and 72 h after irradiation with UVB; ${ }^{*} p<0.05,{ }^{*} p<0.01$, compared with control group; B\&C) Contents of collagen I (B) and collagen III (C) in supernatants of cell culture of skin fibroblasts * $p<0.01$, Compared with control group; D\&E) Contents of MMP1 (D) and MMP3 (E) in supernatants of cell culture of skin fibroblasts $* p<0.01$.

\section{Detection of the expression of type I and III collagen and MMP1 and MPP3 via ELISA}

The cell grouping and senescence induction were the same as those in 1.3.1. The supernatant was collected since the UVB irradiation (at $0 \mathrm{~h}$ ) and at 12, 24, 48 and $72 \mathrm{~h}$ after irradiation. The contents of type I and III collagen and MMP1 and MMP3 in supernatant were determined according to the instructions of ELISA kit, and the results were read using the microplate reader, which was used to reflect the expression of type I and III collagen and MMP1 and MMP3 in senescent cells.

\section{Statistical methods}

SPSS 10.0 software was used for statistical analysis of experimental data. Metrological data were presented as $\bar{x} \pm \mathrm{s}$, and the one-way analysis of variance and paired $t$ test were adopted. $\mathrm{P}<0.05$ suggested that the difference was statistically significant. 


\section{Results}

\section{Preliminary experiment results}

Morphologic observation of skin fibroblasts after in vitro separation and culture: The cells began to climb out from the edge of tissues since the $4^{\text {th }}$ day of tissue explants culture, and the cells were fused at 10-14 d. Porous stone cells could be found in the middle of the long spindle cells, the fused cells received the passage at $1: 3$, and the cell growth accelerated. On the second day, cells could be fused after passage; since the second generation of cells, they showed the typical long spindle shape, and the porous stone cells disappeared.

\section{Expression of P16 protein in senescent skin fibroblasts}

After the irradiation group received the single irradiation of 40 $\mathrm{mJ} / \mathrm{cm}^{2} \mathrm{UVB}$, the expression of P16 protein was significantly higher than that in control group after $12 \mathrm{~h}(\mathrm{P}<0.05)$. The expression of $\mathrm{P} 16$ protein in irradiation group was increased with the prolongation of time, and it reached a peak at $72 \mathrm{~h}$ after irradiation $(\mathrm{P}<0.01)$ (Figure $1 \mathrm{~A})$.

\section{Expression of type I and III collagen and MMP1 and MMP3 in senescent fibroblasts}

Expression of type I and III collagen: After the irradiation group received the single irradiation of $40 \mathrm{~mJ} / \mathrm{cm}^{2} \mathrm{UVB}$, the expression of type I and III collagen was significantly decreased at $12 \mathrm{~h}$ compared with that in control group $(\mathrm{P}<0.01)$; after $24 \mathrm{~h}$, the secretion of type I collagen was basically stagnated, and its content in the supernatant was decreased to less than $10 \%$ of that before irradiation. The secretion of type III collagen was basically stagnated at $72 \mathrm{~h}$, and its content in supernatant was decreased to $5 \%$ of that before irradiation (Figures 1B and 1C).

Expression of MMP1 and MMP3: After the irradiation group received the single irradiation of $40 \mathrm{~mJ} / \mathrm{cm}^{2} \mathrm{UVB}$, the expression of MMP1 and MMP3 was significantly higher than that in control group at $12 \mathrm{~h}(\mathrm{P}<0.01)$; at $72 \mathrm{~h}$ after irradiation, the expression of MMP1 was 4.5 times that in control group and the expression of MMP3 was about 3 times that in control group (Figures 1D and 1E).

\section{Discussion}

Fibroblasts are the main cell components in the dermis of skin, and the collagen fibers, elastic fibers and matrix components secreted by them form the main body of dermis together with fibroblasts [6]. With the increase of age, the skin gradually became senescent. The senescent dermal layer is reflected, at the cellular level, in the fibroblast senescence and decline in its function [7]. Studies have confirmed that the external stimuli can accelerate the skin senescence, such as ultraviolet light, chemical contamination and smoke pollution, among which UV-induced skin senescence has been studied for many years and is easy to be controlled [8]. Therefore, this experiment intended to treat skin fibroblasts with UV to establish the UVinduced skin fibroblast senescence model.

Studies have shown that UV is mainly composed of UVB (wavelength of 280-320 nm) and ultraviolet (UVA) (wavelength of 320-400 nm). Studies [9] have also revealed that UVB radiation can penetrate the skin for $160-180 \mu \mathrm{m}$, and can penetrate the skin layer of human skin and reach the dermis layer, causing skin sunburn and pigmentation, also increasing skin senescence [10]. With the increase in environmental pollution, more and more UVB exposes to the earth's surface gradually, and its damage to the human body is also more serious. Therefore, this study selected UVB to establish the skin fibroblast senescence model to study its damage to skin fibroblasts. The results showed that after 40 $\mathrm{mJ} / \mathrm{cm}^{2}$ UVB was irradiated to human skin fibroblasts for $72 \mathrm{~h}$, the cell growth was stagnated; the cell morphology showed typical senescence (large and flat cells, transparent cytoplasm, and more nuclear particles); $\beta$-gel senescence cell staining showed the strongly positive, and the senescent marker protein P16 was highly expressed, suggesting that UVB successfully induces the human skin fibroblasts senescence.

In addition to the termination of proliferation of skin fibroblasts after senescence, and its corresponding function also significantly changed. It is reported [11] that fibroblasts not only synthesize and secrete collagen, elastin, collagen fibers, reticular fibers and elastic fibers, but also synthesize and secrete glycosaminoglycans, glycoproteins and other matrix components, among which the collagen synthesized and secreted by human skin fibroblasts is important for maintaining the function and features of skin. Studies [12] have shown that the type I and III collagen secreted by human skin fibroblasts not only play a supporting role, but also play an important role in regulating cell proliferation, migration and expression of specific genes. In dermal tissues of human skin, the content of type I collagen is about $80 \%$, and that of type III collagen accounts for about $10 \%$ [13]. Therefore, it is hypothesized that the levels of type I and III collagen were decreased in the skin fibroblast model induced by UVB. It was found in the detection that the levels of type I and III collagen were significantly down-regulated at $12 \mathrm{~h}$ after UVB irradiation and even disappeared at 48 and $72 \mathrm{~h}$; it is confirmed that after the skin fibroblast senescence was induced via UVB, the type I and III collagen secretion was decreased significantly or even disappeared [14].

In UVB induction, the synthesis and secretion of type I and III collagen via skin fibroblasts are lost, accompanied by increased collagen degradation. MMP is a family of zincdependent enzymes encoded by MMP gene, which can specifically degrade the proteins in connective tissues [15]. MMP1, also known as collagenase, can degrade I, III, VII and $\mathrm{X}$ collagen; MMP3, also known as stromelysin-1 or aggrecanase, which can degrade type III, IV, V and X collagen, fibronectin and denatured collagen [16]. In this study, it was found that the expression of MMP1 and MMP3 in skin fibroblasts was significantly increased at $12 \mathrm{~h}$ after the induction of senescence, suggesting that the UVB irradiation of 
skin fibroblasts not only reduces the secretion of type I and III collagen, but also degrades the collagen secreted outside the cells via increasing the expression of MMP.

In conclusion, UVB can induce the skin fibroblasts senescence, whose mechanism may be related to the decrease of collagen synthesis and increase of degradation. Skin fibroblast senescence is the basis of skin senescence [17], and this study laid the foundation for the further research on human skin senescence prevention and treatment.

\section{References}

1. Kim WS, Park BS, Sung JH. Protective role of adiposederived stem cells and their soluble factors in photoaging. Arch Dermatol Res 2009; 301: 329-336.

2. Demirkan NC, Kesen Z, Akdag B, Larue L, Delmas V. The effect of the sun on expression of beta-catenin, p16 and cyclin d1 proteins in melanocytic lesions. Clin Exp Dermatol 2007; 32: 733-739.

3. Helfrich YR, Sachs DL, Voorhees JJ. Overview of skin aging and photoaging. Dermatol Nurs 2008; 20: 177-183.

4. Fisher GJ, Wang ZQ, Datta SC, Varani J, Kang S, Voorhees JJ. Pathophysiology of premature skin aging induced by ultraviolet light. N Engl J Med 1997; 337: 1419-1428.

5. Chainiaux F, Magalhaes JP, Eliaers F, Remacle J, Toussaint O. UVB-induced premature senescence of human diploid skin fibroblasts. Int $\mathrm{J}$ Biochem Cell Biol 2002; 34: 1331-1339.

6. Majora M, Wittkampf T, Schuermann B, et al. Functional conseuences of mitochondrial DNA deletions in human skin fibroblasts increased contractile strength in collagen lattices is due to oxidative stress-induced lysyl oxidase activity. Am J Pathol 2009; 175: 1019 -1030.

7. Wang BX. Skin aging mechanism and progress in research on its prevention and treatment. Chinese Med J 2007; 87: 1375-1376.

8. Yaar M, Gilchrest BA. Photoageing: mechanism, prevention and therapy. Br J Dermatol 2007; 157: 874-887.

9. Hakki SS, Hakki EE, Nohutcu RM. Regulation of matrix metallo- proteinases and tissue inhibitors of matrix metalloproteinases by basic fibroblast growth factor and dexamethasone in periodontal ligament cells J. Periodontal Res 2009; 44: 794-802.

10. Nichols JA, Katiy ar SK, Joi A. Skin photoprotection by natural polyphenols: anti-inflammatory, antioxidant and DNA repair mechaisms. Arch Dermatol Res 2010; 302: 71-83.

11. Fusco D, Colloca G, Lo Monaco MR, Cesari M. Effects of antioxidant supplementation on the aging process. Clin Interv Aging 2007; 2: 377-387.

12. Afanas'ev I. Superoxide and nitric oxide in senescence and aging. Front Biosci (Landmark Ed) 2009; 14: 3899-3912.

13. Fisher GJ, Varani J, Voorhees JJ. Looking older: fibroblast collapse and therapeutic implications. Arch Dermatol 2008; 144: 666-672.

14. Lee YR, Noh EM, Jeong EY. Cordycepin inhibits UVB induced matrix metalloproteinase expression by suppressing the NF-KB pathway in human dermal fibroblasts. Exp Mol Med 2009; 41: 548 -554.

15. Wall IB, Moseley R, Baird DM, Kipling D, Giles P, Laffafian I, Price PE, Thomas DW, Stephens P. Fibroblast dysfunction is a key factor in the non-healing of chronic venous leg ulcers. J Invest Dermatol 2008; 128: 2526-2540.

16. Brenneisen P, Sies H, Scharffetter-Kochanek K. Ultraviolet-B irradiation and matrix metalloproteinases: from induction via signaling to initial events. Ann N Y Acad Sci 2002; 973: 31-43.

17. Moon HJ, Lee SR, Shim SN, Jeong SH, Stonik VA, Rasskazov VA, Zvyagintseva T, Lee YH. Fucoidan inhibits UVB-induced MMP-1 expression in human skin fibroblasts. Biol Pharm Bull 2008; 31: 284-289.

\section{*Correspondence to}

Fuguo Zuo

Department of Dermatology

Shanghai East Hospital

Tongji University School of Medicine

PR China 